\title{
Barking up the Wrong Evidence Tree. Comment on Lurie \& Rosenthal, "Concurrent Partnerships as a Driver of the HIV Epidemic in Sub-Saharan Africa? The Evidence is Limited"
}

\author{
Martina Morris
}

Published online: 9 December 2009

(c) The Author(s) 2009. This article is published with open access at Springerlink.com

It is good that concurrency is becoming a central topic of discussion in the HIV prevention research community [1]. A healthy debate on this topic is long overdue. A productive debate requires a solid understanding of the relationship between behavior (at the individual level) and disease transmission dynamics (at the population level). This relationship determines the relevant evidence. The concepts are simple, but subtle. Get them wrong, and it is easy to make some common mistakes, as Lurie and Rosenthal do here [2].

Lurie and Rosenthal argue that there is not much empirical support for the concurrency hypothesis. While they review a great deal of material, much of it is of limited value, because there is a mismatch between the goal of assessing concurrency and the methodologies used in the studies they review. To understand why, we need to back up a step and ask: Given what we know about how concurrency influences transmission, what is the predicted empirical signature of concurrency's influence on the HIV epidemic?

The short answer is: these dynamics are essentially driven by network effects, so the empirical signature of concurrency is not visible using traditional epidemiological study designs. This is equally true for individual-level and for population-level designs. So we need to design studies differently, and we are just beginning to do that. Until this is done, it is important to avoid some common mistakes.

\footnotetext{
M. Morris ( $\square)$

Department of Statistics, UWCFAR Sociobehavioral and Prevention Research Core, University of Washington, Box 354322, Seattle, WA 98195-4322, USA

e-mail: morrism@u.washington.edu

URL: http://faculty.washington.edu/morrism/
}

At the individual level, armed with the traditional epidemiological approach to relative risk analysis, one might expect to find that concurrency would increase a person's risk of infection, as Lurie and Rosenthal argue in their discussion of the findings from Mattson et al., under the section on "Empirical Evidence". This is the first common mistake. Concurrency is not a risk for the person who has concurrent partners, it is a risk for that person's partners. One way to see this is to consider an initially concordant negative couple, where one of the partners forms a concurrent partnership. The monogamous partner is now exposed to the possibility of disease transmission, not by his/her own behavior, but by the partner's concurrency. For the non-monogamous partner, the risk comes simply from having multiple partners-whether these partners are serial or concurrent is irrelevant, ceteris paribus. Put another way, concurrency increases your risk of transmitting infection, not acquiring it. So the empirical signature is not the correlation between Person A's behavior and status, but between Person A's behavior and their partner's status. Studies that test the association between an individual's concurrency and infection status are theoretically misguided and empirically irrelevant.

A detailed discussion of this issue was published nearly a decade ago, [3] but the issue continues to generate a remarkable amount of confusion in the literature. Papers are routinely published with analyses that use logistic regression to test the significance of a person's concurrency on their own HIV/STI status, and they are cited in the Lurie and Rosenthal comment as empirical evidence that concurrency does not have the predicted effects. The standard approach to relative risk analysis, and the strong tendency towards individualistic reductionism in behavioral research, is so ingrained that this way of thinking is very difficult to change. 
At the population level, armed with the traditional epidemiological approach to ecological analysis, one might expect to find that the prevalence of concurrency in a region would be correlated to the prevalence of HIV, as Lurie and Rosenthal argue in their discussion of the findings from Lagarde et al. [5], under the section on "Empirical Evidence". This is the second common mistake. HIV prevalence is a cumulative measure over timeit represents infections that have accumulated over many years. Concurrency measures on a survey, by contrast, are time-delimited, typically for the last year. The predicted empirical signature of a positive correlation between the two will only be present when both HIV prevalence and behavior have been at equilibrium for some time. That is not the case in any sub-Saharan African country.

This issue, too, was identified in the literature nearly a decade ago, [4] in response to the same Lagarde et al. study that Lurie and Rosenthal cite as support [5]. In contrast to the logic governing individual level effects, which is somewhat subtle, the mismatch between cumulative HIV prevalence and current sexual behavior is fairly obvious. Still, it remains difficult to establish this standard of evidence in the literature.

What does this mean for empirical research on concurrency? It means we need to design studies differently. For an assessment of the impact of concurrency on individual level risk, it means one would need to enroll partners into a study. Ideally, this would be a prospective longitudinal study of incident infection among couples concordant negative at enrollment. The practical and ethical difficulties of such a study are clear, though there have been some valuable public health studies that have sought to follow a chain of transmission (see below). For an assessment of the impact of concurrency on population level risk, HIV incidence needs to be the measured endpoint, not HIV prevalence, with a matching time window for concurrency. This requires a longitudinal community-level design with (controlled or natural) variation in the incidence of concurrency, ideally, an experimental trial that reduced concurrency in an intervention arm. This is a worthwhile endeavor, and formative research has begun in this direction.

In the meantime, there is empirical evidence at the individual level that supports the hypothesis that concurrency increases the transmission of sexually transmitted infections, and that it plays an important role in heterosexual transmission in Africa. Two studies using public health contact tracing data in the US, one on Syphilis [6] the other on Chlamydia, [7] followed up partners and were able to estimate the relative odds of transmitting infection for persons with concurrent partners. Both found that concurrency increased the odds of transmission by more than a factor of three. Partner tracing may not be able to provide the same type of evidence for HIV and concurrency, since the analysis used in these two papers requires identifying the direction of transmission, and that may be hard to establish for prevalent HIV cases. But a 2004 study in Uganda using the BED assay found that among married persons identified as incident cases, $51 \%$ had an infected spouse, while $49 \%$ did not [8]. That implies that about half of the incident cases among married persons may be due to concurrent partnerships. Some fraction of these newly discordant couples will go onto become concordant positive through spousal transmission (as the other $51 \%$ indicates). So the fraction of transmission attributable to concurrency, directly and indirectly, will be higher than $49 \%$.

This kind of partner enrollment is not a feasible option for routine epidemiological studies and surveillance. So if we wish to use more practical traditional survey sampling designs to examine the impact of concurrency at the individual level, there is only one option: the data need to be analyzed using a principled combination of statistical estimation and simulation. Note that it is not possible to represent concurrency explicitly in the traditional compartmental models used for epidemiological simulation. This has been the final methodological obstacle to studying the effects of concurrency. What is needed is "individual based models," with the ability to represent the specific network configurations that represent concurrency.

The methodology needed to use traditional survey sampling data to analyze the impact of concurrency is only now becoming available. Over the last 10 years, our research group has been working to develop statistical methods that provide a principled empirical framework for network epidemiology. These methods are designed to use cross-sectional, egocentrically sampled sexual network data to estimate a model for network dynamics, and then use this model to generate dynamic stochastic complete network simulations. We are releasing the code in a publicly licensed software package for general use [9; see http://www.statnet.org]. Using these methods on US-based data in a recently published paper, we estimate that the small differences in concurrency by race observed in the US are enough to triple the racial disparity in epidemic potential [10]. The underlying contact network we examine in this study affects the transmission of any STI, including HIV, suggesting that concurrency provides a parsimonious explanation for the longstanding racial disparities in the prevalence of all STIs. The more important implication, however, is that this disparity in epidemic potential can be eliminated by a small change-only $5 \%$ of the sexually active population would have to alter behavior, and they would not need to have fewer partners, just have their partners serially rather than concurrently. This is the upside to nonlinear network effects, and we can leverage it for prevention. 
Lurie and Rosenthal make many additional errors of fact, interpretation and omission that are pointed out by the other comments on their piece, but their key argument, that there is no empirical evidence for the impact of concurrency, must be recognized for what it is: the result of a mismatch between the analytic methods used and the process under study, not an assessment of the hypothesis. It is a failure of traditional methodology that is well on the way to being rectified.

Should we therefore wait, as the authors conclude, for better data before developing prevention policies around concurrency? This is the most puzzling of all their assertions. We can argue about whether concurrency is or is not a key determinant of the generalized epidemics in subSaharan Africa, but no one argues that concurrency is irrelevant to transmission. There is nothing to lose, and potentially everything to gain by reducing the prevalence of concurrency, in sub-Saharan Africa, as well as in other populations with disproportionately high rates of HIV/STI. The non-linear nature of connectivity in networks means that small differences in concurrency can create large differences in epidemic potential and thus tremendous opportunities for prevention. It would be a real tragedy if the historical limitations of our research methodology, and the common errors of interpretation that flow from it, were used to justify a do-nothing policy.

Open Access This article is distributed under the terms of the Creative Commons Attribution Noncommercial License which permits any noncommercial use, distribution, and reproduction in any medium, provided the original author(s) and source are credited.

\section{References}

1. Mah TL, Halperin DT. Concurrent sexual partnerships and the HIV epidemics in Africa: evidence to move forward. Aids Behav. 2008. doi:10.1007/s10461-008-9433-x.

2. Lurie MN, Rosenthal S. Concurrent partnerships as a driver of the HIV epidemic in sub-saharan Africa? The evidence is limited. Aids Behav. 2009. doi:10.1007/s10461-009-9583-5.

3. Morris M. Concurrent partnerships and syphilis persistence: new thoughts on an old puzzle. Sex Transm Dis. 2001;28:504-7.

4. Rothenberg R, Potterat J, Gisselquist D. Concurrency and sexual transmission. Aids. 2002;16:678-9.

5. Lagarde E, Auvert B, Carael M, Laourou M, Ferry B, Akam E, et al. Concurrent sexual partnerships and HIV prevalence in five urban communities of sub-Saharan Africa. Aids. 2001;15:87784.

6. Koumans E, Farely T, Gibson J. Characteristics of persons with syphilis in areas of persisiting syphilis in the United States: sustained transmission associated with concurrent partnerships. Sex Transm Dis. 2001;28(9):497-503.

7. Potterat J, Zimmerman-Rogers H, Muth S, Rothenberg R, Green $\mathrm{D}$, Taylor J, et al. Chlamydia transmission: concurrency, reproduction number, and the epidemic trajectory. Am J Epidemiol. 1999;150:1331-9.

8. Mermin J, Musinguzi J, Opio A, Kirungi W, Ekwaru JP, Hladik $\mathrm{W}$, et al. Risk factors for recent hiv infection in Uganda. JAMA. 2008;300:540-9.

9. Handcock MS, Hunter DR, Butts CT, Goodreau SM, Morris M. Statnet: software tools for the representation, visualization, analysis and simulation of network data. J Stat Softw. 2008;24(1).

10. Morris M, Kurth AE, Hamilton DT, Moody J, Wakefield S. The network modeling group. Concurrent partnerships and HIV prevalence disparities by race: linking science and public health practice. Amer J Pub Health. 2009;99:1023-31. 